REDES- Revista hispana para el análisis de redes sociales

Vol.20,\#9, Junio 2010

http://revista-redes.rediris.es

\title{
Capital Social, Cohesión social y uso de la lengua ${ }^{1}$
}

\author{
Carlos Lozares - QUIT, Departamento de Sociologia (UAB) ${ }^{2}$ \\ Mireia Sala - Departamento de Pedagogía (UdG)
}

\section{Resumen}

La lengua (y la escritura) es el vehículo de la mayor parte de interacciones que ocurren en nuestra vida social. Por ello es hacedora, instrumento de comunicación y recurso implícito y/o explícito de las relaciones sociales, y como tal, también del Capital social.

En un colectivo fuertemente cohesivo, en el sentido de gran intensidad de relaciones fuertes internas al mismo en un ámbito dado de la vida social, se puede suponer que la lengua de uso y referencia en dicho ámbito es idéntica en otros ámbitos si el mismo grupo permanece también cohesivo en ellos. Visto a la inversa, el hecho de que en un colectivo se den y usen dos o más lenguas diferentes, desconocidas o con competencias limitadas por partes de dicho colectivo, no facilita la cohesión global del grupo y sí la formación de grupos cohesivos por comunidades lingüísticas. En el caso de la integración, esto es, de relaciones de un colectivo con otros colectivos, identificados como diferentes a través de otros rasgos que no la lengua, se puede suponer que la existencia de una misma lengua de uso y referencia es un mecanismo facilitador, aunque no determinante, de la integración del colectivo en la totalidad de dichos colectivos.

Lo que afirma y trata de explorar este articulo, para distintos colectivos en Catalunya, es por un lado, que la lengua de un colectivo puede ser un factor de homofilia que facilite tanto la cohesión interna como la integración con otros colectivos con la misma lengua. $Y$ por otro lado, que el uso de distintas lenguas no facilita dicha integración en y con los colectivos identificados por otras características.

Palabras clave: Cohesión social - Integración social - Lengua de uso y referencia - comunidad de habla.

\footnotetext{
${ }^{1}$ Este artículo se basa en la investigación cuya referencia es CSO2008-01470, subvencionada por el Ministerio de Educación y Ciencia en el marco del VI Plan Nacional de Investigación Científica, Desarrollo e Innovación Tecnológica 2008-2011. Los autores de este artículo pertenecen a dicho proyecto.

2 Enviar correspondencia a Carlos.Iozares@uab.cat, Centre d’Estudis Sociològics sobre la Vida Quotidiana i el Treball (QUIT), Institut d'Estudis del Treball, (IET). Departament de Sociologia. Universitat Autònoma de Barcelona. 08193. Bellaterra. Cerdanyola del Vallès (Barcelona). Tel: 93.581.2405 / 93.581.1676. Fax: 93.581.2827.
} 
REDES- Revista hispana para el análisis de redes sociales

Vol.20,\#9, Junio 2010

http://revista-redes.rediris.es

\section{Abstract}

Language is a vehicle of interactions that occurs in our social life. So, it is an instrument of communication and resource of implicit or explicit social relationships, and as such, also of social capital.

In a highly cohesive group within the meaning of intensive strong relationships internal to it in a given area of social life, one can assume that the language used and referenced in this field is identical in other areas if the same group remains also cohesive. Viewed conversely, the fact that in a group are given and use two or more different languages, unknown or limited authority in parts of this group, does not provide the overall cohesion of the group and whether the formation of cohesive groups and linguistic communities. In the case of integration, that is, relations of a group with other groups identified as different by other traits that language, it can be assumed that the existence of a common language and reference guide is a facilitating mechanism, though not decisive, the collective integration of all such groups.

Assertions and tries to explore this article, for different groups in Catalonia, is that the language of a collective can be a factor of homophily that facilitates both internal cohesion and integration with other groups with the same language. On the other hand, the use different languages do not facilitate such integration for the groups identified by other characteristics.

Key words: Social cohesion - Social integration - Use and reference language Speaking community. 


\section{Introducción}

La interacción social, y más específicamente el hecho comunicativo, y las relaciones, son componentes y factores básicos de la generación y pertenencia de los hechos sociales. La lengua, con su cara más institucional, y el habla, más pragmática, son contextos, vehículos y recursos que intervienen directamente en las interacciones sociales y ello tanto de una manera objetivada como interiorizada cognitivamente, instrumental como simbólicamente.

Así pues, la perspectiva con que se abordan, en este artículo, las interacciones comunicativas y la lengua no es a partir de las variantes y usos lingüísticos existentes en los distintos grupos sociales de la sociedad catalana, sino a partir de los vínculos que procura la comunicación y relación lingüística y, en un sentido más específico, para hacer corresponder los usos lingüísticos con los grados de cohesión e integración de los diferentes grupos o colectivos que se dan en la sociedad catalana. Más particularmente, se trata de analizar el rol que el catalán y el castellano tienen dentro la sociedad catalana como coadyuvantes o expresiones de la Cohesión e Integración social a partir de determinados colectivos.

Sin pretender descubrir o revisar las reglas que rigen el comportamiento comunicativo de los hablantes en Catalunya, intentaremos clarificar sus tendencias lingüísticas. La intención es la de vincular los cambios lingüísticos de distintos colectivos en Catalunya con el paso o estancia en determinadas instituciones o ámbitos.

En primer lugar, se clarifican los conceptos de Cohesión e Integración social partiendo de la idea de Capital social relacional y sus expresiones de Bonding, Bridging/Linking.

En segundo lugar, se expone la idea teórica de comunidad lingüística para, primero, identificar las distintas comunidades lingüísticas que podemos encontrar en Catalunya y, segundo, contraponerla a la idea de comunidad de habla, que consideramos necesaria para analizar y revisar la realidad interactiva y comunicativa de Catalunya. El bilingüismo de buena parte de la población es un hecho importante que tiene que ver con las relaciones de los individuos y por tanto para entender sus mecanismos de Cohesión e Integración sociales; fenómeno que también sucede a la inversa, esto la influencia de la Cohesión e Itegración sobre el bilingüismo. 
En este sentido, la hipótesis genérica que guía la investigación es que la Cohesión e Integración lingüística, conceptos paralelos pero no idénticos a los de Cohesión social, Integración social, Comunidad lingüística y de habla, se manifiesta de formas distintas según los ámbitos de interacción de los individuos. Sintéticamente, en determinados contextos dónde los lazos suelen ser de carácter afectivo y/o identitario se entiende que la tendencia de todos los colectivos identificados en el estudio van a mantener su lengua de origen, esto es, van a presentar Cohesión lingüística; mientras que en situaciones de lazos o relaciones más formales y/o espontáneos la tendencia de los colectivos va mas allá de preservar su comunidad lingüística, es decir, se verá modificada por las situaciones e amistades de la red, presentando cierta Integración lingüística que, a su vez, repercute a la integración social.

La revisión y análisis de dichas ideas iniciales se ha abordado a través de datos de encuestas egocentradas realizadas a 450 individuos residentes en Catalunya (Barcelona, Sant Feliu del Llobregat y Balaguer) procedentes de distintas oleadas migratorias y autóctonos, a saber: la residente en Catalunya de padres nacidos en Catalunya, la proveniente de otros territorios de España de los años 1950 y 60, la generación que corresponde a sus hijos -nacidos en Catalunya de padres nacidos al resto de España- y finalmente colectivos de la inmigración reciente. ${ }^{3}$

Finalmente, el análisis presenta, a partir de tablas de contingencia, los datos referentes a los cambios lingüísticos de los individuos encuestados según colectivos, vinculándolos con ciertas tendencias de cohesión e integración lingüísticas.

\section{La lengua como mecanismo de cohesión e integración social; lengua, comunidad lingüística y comunidad de habla}

Como ya se ha señalado este artículo se refiere al uso que distintos colectivos hacen de las lenguas y cómo dicho uso puede ayudar a la comprensión del concepto de Cohesión social e Integración social de los mismos colectivos. Para ello empezamos describiendo los conceptos de Cohesión social e Integración social, seguimos con la distinción entre comunidad lingüística i comunidad de habla, para acabar vinculando los cuatro conceptos y exponer qué entendemos por Cohesión lingüística e Integración lingüística.

\footnotetext{
${ }^{3}$ En el apartado metodológico se detalla la información.
} 


\section{La Cohesión social y la Integración social}

En las definiciones clásicas el Capital social guardan entre sí grado elevado de comunalidad semántica pero también algunas diferencias. Se trata de un recurso que proviene $\mathrm{y} / \mathrm{o}$ consiste de la posición en una red de relaciones y que procura reproducción de la posición de dominación o clase (Bourdieu, 1986); un recurso de la estructura de la relaciones que facilita el reconocimiento entre actores, el capital humano y una mayor integración local (Coleman, 1990); o un recurso que proviene de la confianza mutua y reciprocidad y redes entre individuos, colectivos, asociaciones o sociedades (Putnam, 1993, 1995, 2001). El término de redes o relaciones acostumbra a aparece en todas las definiciones.

Desde nuestra perspectiva (Lozares, Verd, López-Roldán, Martí y Molina, 2011) se concibe el Capital social, como la conjunción de dos componentes: el primero, con contenido sustantivo como la confianza mutua, compromiso, normas o valores comunes (Fukuyama, 1995,1997; Portes, 1998, Putnam, 1995, Brehm and Rahn, 1997) y otros contenidos posibles; el segundo, en tanto que forma, como corresponde a la idea de red o estructura relacional de conexiones reticulares para conseguir acceso a distintos recursos (Burt, 1992; Bader, 1990; Knoke, 1999).

Con respecto al componente formal, las relaciones pueden ser hacia dentro del colectivo previamente definido, son las llamadas Bonding. El Bonding se entiende como un recurso de tipo relacional y por tanto como una forma de estructurar las relaciones con contenidos que pueden ser de confianza, reconocimiento mutuo u otros y en general son de carácter expresivo. Pero no sólo existe un único colectivo sino que diversos, por tanto pueden darse interacciones o relaciones entre lo miembros de los colectivos en entre los colectivos como tal. Las relaciones entre colectivos son relaciones hacia fuera o entre individuos de colectivos diferentes. A estas relaciones se denominan Bridging; tienden a aportan nuevas fuentes de información o innovación representando oportunidades para los agentes y de naturaleza más instrumental (Johanson, 2001).

Para definir la Cohesión e Integración social se han de considerar también, como en el Capital social, dos componentes necesariamente vinculados entre sí: el primero, es el componente formal basado en las relaciones; el segundo, es el contenido que puede corresponder a los indicados para el Capital social. Para la Cohesión social reservamos las relaciones de tipo Bonding dentro del colectivo en que se identifica la Cohesión; para la Integración social, para la que se consideran dos o más colectivos tenemos en cuenta las relaciones Bridging, hacia fuera, externas o entre colectivos. Así pues ambos conceptos se entienden como formas de distribución o 
de estructurar el Capital social (ver Lozares, Verd, López-Roldán, Martí y Molina, 2011).

\section{Comunidad lingüística y comunidad de habla}

La comunidad lingüística la entendemos como un colectivo (sociedad, pueblo, etc.) de personas que comparten una misma lengua de origen. Por tanto, se trata de un colectivo al que se le supone el dominio del sistema de una lengua, un asentamiento en un espacio territorial determinado y una cierta identificación y cohesión en tanto que comunidad por tener esa misma lengua de origen y desarrollarla como proceso de comunicación natural entre sus miembros. Dicha lengua es común y caracteriza las personas de la comunidad lingüística, independientemente del uso que hagan esas personas de otras lenguas en determinadas situaciones. La comunidad lingüística es pues un colectivo con un idioma de origen común que participa en una impronta "indeleble" a partir de un proceso natural de comunicación. Por comunidad de habla se entiende una población o colectivo que se comunica a partir de un idioma en el marco de acontecimientos, instituciones, momentos o situaciones específicas de la vida social; es decir, es de carácter más situacional y circunstancial. El concepto se enmarca en la etnografía de la comunicación de mediados del siglo XX con Labov (1972), Gumperz (1968), Gumperz y Hymes (1972) que se centraron en la variación social del lenguaje o uso de la lengua según espacios y grupos sociales de pertenencia y/o situaciones de vida. El concepto, al conjugar la palabra comunidad con la de habla remarca el hecho interactivo del uso de una determinada lengua como habla, esto es, de situación pragmática y contextual como oposición a la lengua como institución. Este uso de la lengua producida desde una vertiente más pragmática y relacional define pues la comunidad de habla a partir de su uso real para las personas de un colectivo definido que, al menos en dichas situaciones, el habla estructura y desarrolla su vida social. Por tanto, comunidad de habla es, desde el punto de vista aquí adoptado, un colectivo o conjunto de individuos que se comunican, interaccionan y relacionan en la misma lengua en determinados momentos o situaciones de la vida social.

Haciendo un balance entre ambos conceptos y siguiendo a Duranti (1997), la comunidad de habla es el resultado de las actividades comunicativas que lleva a cabo un determinado grupo de personas, diferenciándola así del concepto chomskiano de comunidad lingüística, que remite al conjunto de hablantes de una misma lengua $\mathrm{y}$, además, concibe ese conjunto como una comunidad ideal $\mathrm{y}$ homogénea. Así, siendo condición necesaria, pero no suficiente, el conocimiento y competencia de un idioma para establecer un colectivo como comunidad lingüística 
o como comunidad de habla, ya que para el primero ese idioma tiene que ser además lengua de origen (Mollà, 2002) y para el segundo ha de ser usado como medio de comunicación entre los miembros del colectivo en determinadas situaciones; ambas comunidades pueden cohabitar en un mismo territorio. Una comunidad de habla puede estar formada por personas de distintas comunidades lingüísticas o una comunidad lingüística ser también comunidad de habla si en una o en todas las situaciones tal lengua es la usada en los intercambios comunicativos. En este sentido, un colectivo que es una comunidad lingüística puede pertenecer a una, dos o más comunidades de habla según situaciones, momentos, instituciones o ámbitos de la vida.

En el caso catalán, en el que dos lenguas, catalán y castellano son conocidas y usadas por gran parte de la población se pueden dar varias situaciones de coexistencia dónde los límites de comunidad lingüística y de habla devienen más porosos y borrosos con intensidades diversas y dónde el bilingüismo juegue un papel central en la integración.

\section{El bilingüismo como balance entre la Cohesión y la Integración lingüística}

Si la cohesión interna de una comunidad lingüística se refuerza en función de la densidad de las relaciones, transacciones o comunicaciones internas a dicha comunidad (Mollà, 2002), podemos retomar la idea de Cohesión social e Integración social para relacionarlas con la Cohesión lingüística e Integración lingüística y ver el papel que juega el bilingüismo como balance entre las dos.

La cohesión de una comunidad lingüística, esto es la Cohesión lingüística, se entiende como la existencia de interacciones a partir del uso y práctica interna al colectivo de su propia lengua. En cambio, cuando hablamos de la integración de una comunidad lingüística o Integración lingüística de un colectivo entendemos que la existencia de estas interacciones se dan entre dos o más comunidades lingüísticas usando una de las lenguas de origen o una tercera lengua aprendida por las distintas comunidades, ya sea en la totalidad de su vida social, ya sea en sectores de la misma. En este sentido, y aunque el cambio en las comunidades lingüísticas depende en gran medida del grado de apertura y capacidad integradora de la comunidad lingüística de acogida y de llegada, acostumbran a ser las razones instrumentales las que más determinan en qué situaciones, momentos o instituciones se usa una u otra lengua. Así, la Integración lingüística parcial o total de dos comunidades depende del tiempo de adquisición de la segunda lengua, del estatus, prestigio o de las funciones sociales atribuidas a cada una de las lengua (Weinreich, 1974); esto es, para darse la Integración lingüística unas y otras 
comunidades lingüísticas tienen que considerar como un bien social positivo y conveniente la utilización de otra lengua diferente a la propia, más que identificarse con razones o otros factores simbólicos, patrióticos o nacionales.

Si sólo existe una sola comunidad lingüística en un territorio sin que sea objeto de implicación de las comunidades lingüísticas de otros territorios no tiene por qué tener conciencia, y menos identitaria, de que la lengua sea un elemento de Cohesión social, pues el contacto o interacción entre individuos va de sí. Tampoco se da, en dicho caso, la presencia de comunidades de habla en situaciones o ámbitos de vida en coexistencia con otras comunidades lingüísticas, ni se plantea la necesidad de integración, pues es la única comunidad lingüística y de habla. En este caso, cualquier factor de Cohesión social reforzaría la Ccohesión lingüística y viceversa. La comunidad lingüística coincidiría con la comunidad de habla en todas las situaciones y ámbitos de vida, si bien podrían darse grupos o colectivos más o menos cohesionados por otros factores o tipos de relaciones.

Por otro lado, partiendo de la existencia de distintas comunidades lingüísticas en un territorio, si la Integración lingüística fuese tal que en todas las situaciones se utilizara la lengua propia de una de las comunidades lingüísticas para las interacciones comunicativas, la Integración lingüística y Integración social podrían ir parejas retroalimentándose mutuamente $y$, probablemente, en detrimento de la Cohesión lingüística absoluta de y en cada una de las comunidades lingüísticas y subsidiariamente de sus Cohesiones sociales respectivas.

Por tanto, como posibilidades imaginadas se pueden dar formas en que comunidades lingüísticas diferentes queden cerradas sobre sí y segmentadas sin ninguna comunicación mutua ni espacios o momentos para la existencia de comunidades de habla o, por el contrario, se pueden dar formas de Integración lingüística en la que una lengua de una comunidades lingüísticas es el vehículo para las otras. Pero ni la máxima clausura, ni la total apertura de un colectivo son realistas ni necesarias para un mayor beneficio del grupo, esto es, para una mayor distribución de su Capital social. En consecuencia, para que el Capital lingüístico revirtiera en el Capital social y viceversa, tendríamos que preguntarnos acerca de lo que puede ser más conveniente y benéfico para las comunidades lingüísticas: ¿una fuerte cohesión interna de cada comunidad social o lingüística de origen o la integración total lingüística a partir de una sola lengua -sea de una o de otra comunidad lingüística-dejando la otra en residuo?; ¿la subsistencia de comunidades lingüísticas con espacios y ámbitos sociales para que se den comunidades de habla de alguna de las lenguas de origen? $Y$ en cada caso ¿cuándo, dónde o por qué mecanismos? 
Las cuestiones precedentes que no son simples lo son menos cuando, como es el caso de Catalunya, se da la situación de bilingüismo en una buena parte de dos de las comunidades lingüísticas más importantes, la comunidad de catalanohablantes y la de castellanohablantes. Las dos comunidades usan, sea en todos los ámbitos de la vida social, sea en algunos específicos, una u otra lengua según momentos y situaciones. Así, en Catalunya las funciones atribuidas a cada lengua pueden estar más superpuestas y/o retroalimentadas siendo más o menos explicativas, dados los intercambios generacionales, interacción en la escuela, la integración real en otros aspectos de la vida social diferentes a la lengua o la co-oficialidad del catalán y el castellano. Dicho bilingüismo puede jugar un papel clave en el equilibrio entre Cohesión lingüística e Integración lingüística, revirtiendo en cierto modo a la Cohesión social e Integración social de los distintos colectivos. Por lo tanto, la pregunta es acerca de los espacios y/o ámbitos en que cada comunidad lingüística usa una u otra lengua. Se trata pues de analizar, para las distintas comunidades lingüísticas en Catalunya, tanto el mantenimiento o perpetuación de las tales comunidades y/o su mixtura así como el grado de Cohesión e Integración lingüísticos y también sociales.

\section{El planteamiento y las hipótesis de investigación}

Los conceptos de Cohesión e Interacción social tienen tanto que ver con la idea de comunidad lingüística como con la de comunidad de habla. Ambos conceptos se inscriben en el binomio de sociedad y lengua pues se vinculan, por un lado, a la composición social de la sociedad y, por otro, a la utilización de la lengua como identidad e instrumento en dichos colectivos cohesivos y/o integrados. Los procesos interactivos intervienen en la constitución de grupos y colectivos más o menos cohesivos y el dicha interacción la lengua y el hablar son parte y actores; dichos procesos los son también de las estructuras y dinámicas socioculturales de la sociedad pues las generan y consolidan. Dentro de los procesos interactivos los del habla y lengua son también reflejo y factor de identidad de un colectivo y más extensamente de una sociedad. Por lo tanto, la Cohesión social de una comunidad lingüística, esto es, la existencia de interacciones internas en el colectivo de la misma lengua de origen y, la Integración lingüística entre comunidades a partir de una de las dos lenguas de origen, depende y puede explicarse por distintos procesos. Aquí sólo se intenta examinar qué situaciones, espacios sociales o instituciones favorecen con mayor probabilidad el uso de una de las dos lenguas oficiales en Catalunya (catalán y castellano). Traducido en Cohesión social e Integración social es cuestión de examinar si un colectivo global o una parte del mismo que conforma una comunidad lingüística, por ejemplo con lengua de origen 
no oficial, bien se mantiene cohesivo y cerrado en su propia comunidad lingüística, presentando Cohesión lingüística en el conjunto de su vida social o bien se inicia y desarrolla un proceso de integración con otra comunidad lingüística, esto es, presenta Integración lingüística generando o desarrollado una comunidad de habla con una de las lengua oficiales según circunstancias o instituciones.

En el artículo los colectivos de entrada corresponden a comunidades lingüísticas y se examinan las relaciones que mantienen con personas de un colectivo idéntico. Estas relaciones pueden ser más o menos intensas; es la parte formal de la Cohesión social. Las relaciones son de colectivos Egos, comunidades lingüísticas con sus elegidos Alteri de la misma comunidad de los Egos. Son pues relaciones con colectivos equivalentes; por eso hablamos de relaciones de Cohesión social en dicho colectivo. Es algo similar al concepto de Homofilia aunque no coincidan exactamente ambos conceptos. El contenido de las relaciones se refiere a relaciones de trato y conocimiento mutuo; sobre dicha relación se superpondrá la relación del habla en determinadas situaciones.

Lo que interesa analizar son las situaciones, espacios o ámbitos de la vida social en los que se da, se facilita o se favorece el tránsito, o sea la integración, de una comunidad lingüística a otra. Esto es, se trata de revisar la realidad cohesiva y/o integradora de distintos colectivos en Catalunya a través del uso que ellos tienen de la lengua en tanto que comunidades lingüísticas y de habla.

Se parte del supuesto de que la interacción lingüística es una práctica que influye notablemente en una mayor cohesión interna intragrupo y/o de integración entre comunidades lingüísticas a partir de la flexibilidad que aporta el bilingüismo y su potencialidad como generador de comunidades de habla. La pregunta de investigación es la siguiente, ¿en qué contextos, situaciones, momentos e instituciones los individuos de una comunidad lingüística que mantienen un cierto nivel de Cohesión social a partir de las relaciones con sus conocidos y con los que se comunica en la misma lengua de origen, se comunican e interactúan con otros de otra comunidad lingüística en una lengua que no es la propia? Es decir ¿en qué contextos, situaciones, momentos y en qué lengua los individuos caracterizados como una comunidad lingüística interactúan con alguna de las lenguas propias de una de las comunidades a partir del fenómeno del bilingüismo generando comunidades de habla y a su vez cierta Integración lingüística que se alejaría del cierre, como mínimo lingüístico, de dicho colectivo? 
Con respecto al uso que los individuos hacen de las lenguas en situación, momentos o espacios sociales se presentan dos posibilidades. Por un lado, si los individuos que provienen de una comunidad lingüística mantienen el uso de su lengua de origen en distintos ámbitos podemos hablar igualmente de comunidades de habla, siendo la comunidad de habla igual a la comunidad lingüística. Pero si existen cambios, esto es, variaciones en el uso según contextos, se hablará de procesos o cambios hacia una "catalanización" si la lengua usada en la comunidad de habla del contexto seleccionada es el catalán y un proceso de "castellanización" si la lengua de InL o de comunicación entre distintas comunidades lingüísticas en cierto ámbito pasa a ser el castellano.

El bilingüismo es una situación de balance entre comunidad lingüística y comunidad de habla. Si se parte de un bilingüismo instrumental en el que predominan, aunque no exclusivamente, las razones de poder, prestigio y/o legales de una lengua con respecto a la otra se puede suponer que en situaciones de intercambio formales e institucionales las comunidades de habla se generaran y manifiestan en torno a la lengua catalana, pues existen mecanismos $\mathrm{y} / \mathrm{o}$ procedimientos institucionales $\mathrm{y} / \mathrm{o}$ legales con tendencia y finalidad integradora, al menos lingüística, en la mayoría de instituciones o empresas del ámbito catalán. Por contra, se tenderá a usar la lengua de origen en situaciones afectivas, de lazos fuertes e identitarios, como es en el núcleo familiar o de ocio, reforzando así los lazos de la comunidad lingüística y, por tanto, la Cohesión lingüística que, a su vez, al coincidir con grupos previamente definido repercute en su Cohesión social.

Por otro lado, el uso de una u otra lengua no sólo depende de las situaciones o ámbitos de la vida social sino de las relaciones de Cohesión y/o Integración social entre los individuos. El fenómeno y situación de intercambio lingüístico se producirá en mayor grado entre individuos de las comunidades lingüísticas catalana y castellana gracias a que las relaciones sociales se facilitan por su bilingüismo, lo que no será el caso con o ente otras lenguas que no sea el catalán y/o castellano por la inexistencia de tal bilingüismo. Esto es, podrá existir Integración lingüística en alguna medida, para individuos de comunidades lingüísticas de lengua no oficial en Catalunya, si es que da algún tipo de Integración social que provenga de razones instrumentales o expresivas, como es el paso por la escuela, las amistades o el tipo de trabajo. En otras palabras, en la medida que los individuos de comunidades lingüísticas de lenguas no oficiales en Catalunya presenten otros mecanismos de Integración social tales como las relaciones de amistad con otros colectivos su Integración lingüística también se verá reforzada. 
Por lo tanto, los individuos presentan una mayor Cohesión lingüística con respeto a su lengua de origen con colectivos que tengan perfiles semejantes al suyo, mientras que los que se relacionen con individuos de colectivos distintos su Integración lingüística se va a dar en mayor medida hacia el catalán, en ámbitos formales, o hacia el castellano, en ámbitos menos institucionalizados. Dicha Integración lingüística en entornos no familiares es más alta entre aquellos que su lengua de origen no corresponde a ninguna de las dos lenguas oficiales; por este motivo, y a la inversa, los que si tienen como lengua de origen una de las oficiales van a presentar menos Integración lingüística.

Enumeramos y concretamos las hipótesis de la investigación en las siguientes

1. En las relaciones con los Alteri, los colectivos de una misma comunidad lingüística, presentan una mayor tendencia cohesiva con los Alteri de su propio colectivo. Esta cohesión será mayor en las comunidades lingüísticas que se sienten asentadas al territorio.

2. Genéricamente en contextos de lazos fuertes y afectivos y con más sentimiento identitario, la tendencia de las comunidades lingüísticas entre los Egos es la de mantener su lengua de origen; esto es, se espera que la comunidades lingüísticas sean en dichos contextos comunidades de habla. Es posible que esa tendencia se dé con menor intensidad en las segundas generaciones de olas migratorias. Así, en contextos de lazos fuertes y afectivos la tendencia va ser la de mantener la lengua de origen más que presentar procesos de "catalanización" o "castellanización" en el habla.

3. Tratándose de procesos de transferencia lingüística, esto es, de generación de comunidades de habla entre colectivos de distintas comunidades lingüísticas, se puede suponer que el uso del catalán, más que el uso del castellano, será más utilizado en situaciones o entornos de trabajo, esto es, en entornos de relaciones formales y lazos instrumentales.

4. En los contextos de ocio se tenderá al proceso de utilización del castellano, pues los catalanohablantes se les sabe bilingües, mientras que las nuevas olas de migrantes pertenecen a comunidades lingüísticas de habla castellana o se les atribuye este hecho. Los otros dos extremos, el uso del catalán o el uso de otra lengua en dichos contextos se explicaría por una casi total relación en la red con Alteri de su mismo origen y comunidad lingüística. 


\section{Metodología}

Los datos que nos han permitido trabajar esta temática proceden del proyecto I+D+I con referencia CSO2008-01470, titulado "Estudio comparado de casos sobre la influencia mutua entre capital e integración sociales y la inserción, estabilidad, promoción y cualificación en el empleo (CASREDIN)", siendo Carlos Lozares el investigador principal y subvencionado por el Ministerio de Educación y Ciencia dentro del marco del VI Plan Nacional de Investigación Científica Desarrollo e Innovación Tecnológica 2008-2011.

La muestra seleccionada, metodología y el tipo de cuestionario se detallan en el artículo de Lozares, López, Verd, Marti, Bolibar y Cruz, (2011). Resumiendo, la muestra de Egos a entrevistar ha sido aleatoria y estratificada. La estratificación, con dos etapas, se ha hecho primero según localidades de residencia de Ego y, segundo, según colectivos de población que responden a un análisis factorial realizado sobre el censo de Catalunya del 2001 (López-Roldán y Lozares, 2008)

Las localidades seleccionadas han sido Barcelona, Sant Feliu del Llobregat y Balaguer y los colectivos se han seleccionado según edad y corresponden a dos oleadas migratorias y a la población autóctona: a) individuos mayores de 55 años que hayan nacido en Catalunya de padres nacidos en Catalunya ( $\mathrm{NC}+55 \mathrm{pNC})$; b) individuos de entre 25 y 55 años que hayan nacido en Catalunya de padres nacidos en Catalunya (NC25-55pNC); c) individuos mayores de 55 años nacidos en el resto de España $(\mathrm{NE}+55)$; d) individuos de entre 25 y 55 años de padres nacidos en el resto de España (NC25-55pNE); e) individuos mayores de 25 años nacidos en Ecuador (NEcuador) y f) individuos mayores de 25 años nacidos en Marruecos (NMarruecos).

Los datos consisten en 450 encuestas de redes personales con 4 bloques de principales: un primer bloque de preguntas de caracterización socio-demográfica de los Ego, la pregunta generadora de redes de 30 Alteri, un tercer bloque de preguntas de caracterización socio-demográfica de cada Alter mencionado y las relaciones existentes entre cada par de Alteri.

De las preguntas socio-demográficas hemos seleccionado aquellas en las que Ego hace referencia, primero, a su lengua de origen y a su colectivo, segundo, al uso que él hace de la lengua en distintos ámbitos: el familiar, el laboral y el de ocio; con las posibilidades de respuesta: a) catalán, b) castellano, c) catalán y castellano por igual $y \mathrm{~d}$ ) otra lengua. $\mathrm{Y}$, un segundo volumen de datos nos los han proporcionado las características socio-demográficas de los Alteri que conoce Ego. 
El conjunto de individuos encuestados representa orígenes y estancias temporales distintas en Catalunya que pueden conllevar, en términos de CoS e InS, diferencias sociales en estatus, categoría social, mercado laboral, nivel educativo, además de otras características como hábitos, modos de vida e idioma. Es precisamente en este último sentido, las características idiomáticas, por las que nos ha interesado redefinir los grupos de base.

La matriz inicial se ha reducido un $11,1 \%$, pasando de los 450 encuestados iniciales a los 385 individuos que se distribuyen según los colectivos iniciales, después de aplicar los criterios que siguen, como muestra la tabla 1 :

a) De los nacidos en Catalunya de padres nacidos en Catalunya mayores de 55 años y los nacidos en Catalunya de padres nacidos en Catalunya entre 25 y 55 años, se han seleccionado los que además tienen como lengua de origen el catalán.

b) En el mismo sentido, se ha establecido un segundo grupo por los mayores de 55 años que han nacido en el resto de España y además tienen como lengua de origen el castellano.

c) El tercer grupo se ha definido con los que han nacido en Catalunya entre 25 y 55 años de padres nacidos en el resto de España y que se autodefinen como bilingües de origen o con el castellano como lengua de origen.

d) Los dos últimos grupos, que corresponden a dos colectivos de la nueva oleada migratoria se han definido a partir del origen ecuatoriano y el origen marroquí, eliminando los que no tienen como lengua de origen el castellano o otra lengua 1 , respectivamente.

\section{Comunidades lingüísticas según colectivo}

\begin{tabular}{|c|c|c|c|c|c|}
\hline \multirow[t]{2}{*}{ Origen Ego } & \multicolumn{5}{|c|}{ Lengua de origen de Ego } \\
\hline & Catalán & Castellano & $\begin{array}{l}\text { Ambas por } \\
\text { igual }\end{array}$ & Otra, árabe & Total \\
\hline NC+55pNC & 64 & 0 & 0 & 0 & 64 \\
\hline NC25-55pNC & 53 & 0 & 0 & 0 & 53 \\
\hline NC25-55pNE & 0 & 62 & 6 & 0 & 68 \\
\hline NE+55 & 0 & 61 & 0 & 0 & 61 \\
\hline Ecuador & 0 & 66 & 0 & 0 & 66 \\
\hline Marruecos & 0 & 0 & 0 & 73 & 73 \\
\hline Total & 117 & 189 & 6 & 73 & 385 \\
\hline
\end{tabular}

Tabla 1. Tabla de contingencia entre origen de Ego y lengua de origen de Ego.

La intención de la reducción de la matriz es la de vincular cada colectivo con una comunidad lingüística. La razón obedece que en dichos colectivos, entendidos ahora como comunidades lingüísticas, se puedan conocer las relaciones de comunicación y conocimiento independientemente de la lengua $y$, segundo, se pueda conocer la 
lengua que en determinadas situaciones usan dichos encuestados Egos, es decir, se puede conocer si forman comunidades de habla.

Por lo tanto, la reducción de la matriz ha supuesto incorporar a los colectivos previamente definidos la dimensión lingüística, hecho que nos permitirá analizar sus grados de Cohesión social e Integración social genérica a partir de las relaciones de conocimiento y comunicación con sus Alteri y comprobar el comportamiento lingüístico entre los Egos de dichos colectivos según algunos contextos de su cotidianidad, estos son, en la familia, en el trabajo y con los amigos.

\section{El análisis. La Cohesión e Integración entre los Egos de las comunidades lingüísticas y sus conocidos Alteri}

El análisis se presenta en dos bloques. Primero, se examina si los Egos encuestados de las diferentes comunidades lingüísticas presentan más bien Cohesión social o Integración social respeto a los Alteri de su misma naturaleza. Segundo, suponiendo que se da cierto grado de Cohesión social en las comunidades lingüísticas, se analiza el comportamiento de los Egos en distintas situaciones o ámbitos de su cotidianidad, esto es, para comprobar si se generan comunidades de habla distintas a la comunidad marcada por su origen.

En primer lugar, al cruzar en una tabla de contingencia el origen o colectivo de pertenencia de los Egos con el colectivo de pertenencia de los individuos con los que ser relaciona, esto es, con sus Alteri; vamos a revisar si partiendo de unos colectivos base que hemos equiparado a distintas comunidades lingüísticas existe Cohesión social o más bien cierta Integración social entre los colectivos. Para revisarlo se ha de tener en cuenta que la diagonal del a tabla de contingencia va a marcar las relaciones de cada colectivo con los Alteri del mismo colectivo, es decir, nos marca el porcentaje de Cohesión social, mientras que los valores que se alejan de esta diagonal nos marcan los porcentajes de Integración social para cada par de colectivos.

La tabla 2 nos muestra la tendencia cohesiva para los colectivos o comunidades lingüísticas a partir del conocimiento y comunicación con los alteri que son de su misma comunidad lingüística. En naranja se marca la diagonal cohesiva y en rojo las integraciones respectivas, siendo menos intenso cuando menor es el porcentaje de integración.

Vemos que todos los colectivos tienden a relacionarse más con Alteri de su mismo origen $y$, por ende, naturaleza lingüística. Se configuran y reafirman ahora cohesivamente las descritas comunidades lingüísticas de los catalanes nacidos a 
Catalunya, mayores y menores de 55 años, pues cerca del $80 \%$ de la naturaleza de sus Alteri se asemeja a la suya.

En relación al colectivo ecuatoriano y marroquí, la tendencia es la misma acerca de la cohesión con sus Alteri de su mismo origen geográfico y con la misma lengua de origen, hecho que nos permite identificarlos como dos comunidades lingüísticas que en sus relaciones refuerzan su Cohesión lingüística y social. No obstante estas dos comunidades destacan por reconocer en su red de alteri, catalanes de padres catalanes más que catalanes nacidos en el resto de España o de nacidos en Cataluña de padres nacidos en el resto de España, pues podrían atribuir al hecho de saber catalán su origen también catalán (cabe recordar que las características de los Alteri las proporcionan los mismos Egos).

De igual interés es el hecho que el colectivo ecuatoriano presente una mayor integración con colectivos de otros orígenes, siendo una explicación posible la interacción y relación de estos con otros colectivos de origen sudamericano con la misma lengua de origen que los ecuatorianos.

En cambio, los colectivos o Egos caracterizados como primera oleada migratoria y, sobre todo sus hijos/as potenciales ya nacidos en Catalunya de padres que, aunque residentes en Catalunya, nacieron en el resto de España; presentan cierto grado de InS, esto es, se relacionan con Alteri de naturaleza distinta a la suya con mayor porcentaje que los otros colectivos, concretamente con los catalanes de origen se relacionan a la par que con los de su mismo colectivo, siendo el porcentaje de relaciones hacia los catalanes de origen del $38,1 \%$ y con los de su mismo colectivo del 34,5\%. De todas formas estos porcentajes pueden presentar un cierto grado de espejismo si no se miran los porcentajes marginales en filas como criterio comparativo. Por ello se recurre a los residuos ajustados en donde se amortigua el efecto manteniéndose el efecto comparativo (ver tabla 3 ). 


\begin{tabular}{|c|c|c|c|c|c|c|c|}
\hline Origen Alteri & $\mathrm{NC}+55 \mathrm{NpnC}$ & $\begin{array}{l}\text { NC25- } \\
55 N C p n C\end{array}$ & NC25-55pnE & $N E+55$ & NEcuador & NMarruecos & Total \\
\hline $\begin{array}{l}\text { NC }+55 p n C \\
\text { NC25-55pnC }\end{array}$ & $80,5 \%$ & $76,4 \%$ & $38,1 \%$ & $26,8 \%$ & $12,9 \%$ & $10,5 \%$ & $39,1 \%$ \\
\hline NC25-55pnE & $8,7 \%$ & $14,0 \%$ & $34,5 \%$ & $21,4 \%$ & $4,7 \%$ & $4,5 \%$ & $14,5 \%$ \\
\hline$N E+55$ & $9,2 \%$ & $5,0 \%$ & $22,7 \%$ & $49,4 \%$ & $2,4 \%$ & $5,0 \%$ & $15,4 \%$ \\
\hline NEcuador & $0,1 \%$ & $0,1 \%$ & $0,1 \%$ & $0,2 \%$ & $65,9 \%$ & $0,9 \%$ & $11,5 \%$ \\
\hline NMarruecos & $0,1 \%$ & $0,3 \%$ & $0,0 \%$ & $0,2 \%$ & $0,5 \%$ & $73,5 \%$ & $14,1 \%$ \\
\hline Otro lugar & $1,5 \%$ & $4,3 \%$ & $4,5 \%$ & $2,0 \%$ & $13,6 \%$ & $5,6 \%$ & $5,3 \%$ \\
\hline Total & & & & & & & \\
\hline
\end{tabular}

Tabla 2. Caracterización de la cohesión e integración de las comunidades lingüísticas (en porcentaje).

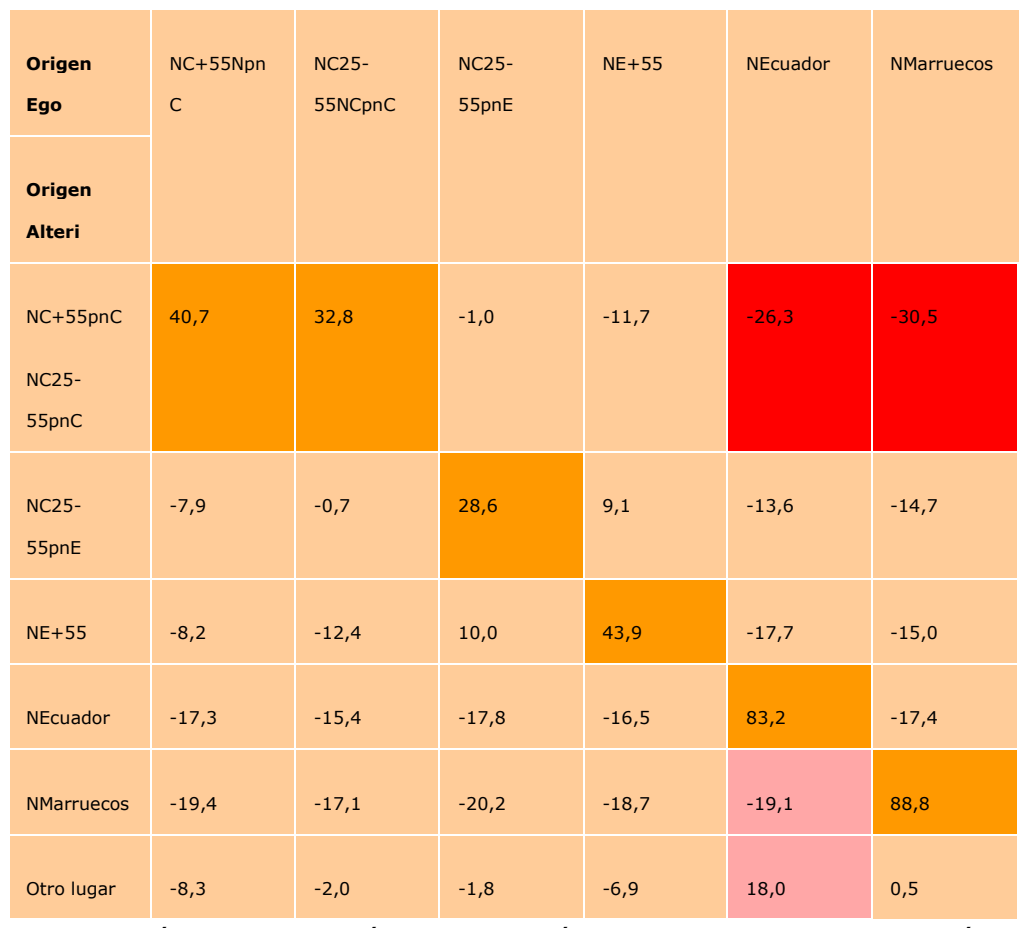

Tabla 3. Caracterización de la cohesión e integración de las comunidades lingüísticas (residuos corregidos).

Los límites de las comunidades lingüísticas que parecen algo borrosos con relación a la cohesión e integración, en particular en los colectivos que se refieren a la primera oleada migratoria, se difuminan aún más cuando observamos el uso habitual que estas comunidades hacen de su lengua en el contexto familiar, laboral y de ocio. Precisamente, la segunda parte del análisis trata de examinar qué pasa en el uso del habla en estos ámbitos en los que los Ego de una comunidad interaccionan con otras comunidades es espacios de lazos más o menos afectivos. 
Se ha de recordar que en dichos ámbitos, espacios o instituciones, el uso de la lengua de origen de Ego puede mantenerse 0 cambiar, presentando "catalanización" o "castellanización".

En concreto, en las interacciones en distintos ámbitos nos podemos encontrar con Egos que siendo de una comunidad lingüística pase a usar una lengua distinta a su lengua original, generando comunidad de habla, gracias y a través del bilingüismo y integrándose, como mínimo lingüísticamente a otra comunidad. Los colectivos o comunidades lingüísticas representadas por Egos pueden manifestar los siguientes cambios:

1) Estabilidad en el "catalán", cuando la lengua de origen y la de habla usada en el ámbito estudiado es el catalán.

2) Estabilidad en el "castellano" si lo es en castellano.

3) Mantenimiento en la situación "Mixta", cuando tanto de origen como en el ámbito concreto Ego se ha definido como bilingüe de catalán y castellano.

4) "Catalanización", cuando Ego procede de una comunidad lingüística distinta a la catalana y pasa al catalán como lengua de uso en algún ámbito o espacio concreto de su vida social.

5) "Castellanización", si sigue el mismo procesos anterior con respecto al castellano.

6) "De Otras comunidades lingüísticas (en particular árabe) a Mixto" si pertenece a una comunidad lingüística distinta a una de las dos lenguas oficiales y se pasa al uso del catalán y/o castellano indistintamente. ${ }^{4}$

Veamos ahora como se manifiestan estos cambios en el habla de los colectivos o comunidades lingüísticas definidas a partir de Ego en tres ámbitos distintos: el contexto familiar, de lazos afectivos e identitarios, el contexto laboral, de lazos formales y débiles y, por último, el contexto de amistades y ocio de lazos más o menos fuertes y de generación espontánea.

\section{En situación de contexto familiar}

La tabla 3 representa las tendencias en \% del comportamiento en el ámbito familiar entre los Egos entrevistados de los diferentes colectivos o comunidades lingüísticas

\footnotetext{
${ }^{4}$ No se ha considerado el proceso de catalán o castellano hacia otra lengua porque entre los Egos encuestados no se ha dado el caso.
} 
en su tránsito a comunidades de habla. Los procesos de cambio o mantenimiento se han de entender con referencia a su lengua de origen o su comunidad lingüística, partiendo de esta establecemos las comunidades de habla en el contexto familiar y revisamos cuanto mantenimiento o cambio se presenta para cada colectivo de Ego. En verde oscuro se marca la tendencia estática, de mantenimiento de la lengua en el ámbito afectivo; mientras que en rojo se visualizan los procesos de cambio.

En referencia a este contexto la tendencia general para todos los colectivos de Ego o comunidades lingüísticas es la de mantener la lengua de origen, particularmente fuerte o completa en los Egos de la comunidad lingüística de catalán, el $96 \%$, el $95,9 \%$ la marroquí, y en $98 \%$ la ecuatoriana. Baja un tanto este uso en los Egos de la primera ola migratoria: un $\mathbf{8 6 \%}$ de los nacidos en el resto de España siguen utilizando el castellano, mientras que para los nacidos en Catalunya de padres catalanes nacidos en el resto de España lo hacen en un $75 \%$; por lo tanto en esta comunidad $y$ en el contexto más afectivo e identitario se da un $17 \%$ de catalanización. Este proceso no se da a la inversa, esto es, el porcentaje de nacidos en Catalunya que pasan, en el ámbito familiar, al uso del castellano siendo su comunidad lingüística el catalán sólo es del 3,1 y 3,8; respectivamente.

En el contexto y situación familiar se da un proceso mayor de catalanización que de castellanización. Los Egos de origen catalán tienden a cambiar menos de lengua en dicho contexto, mientras que para los de origen castellanohablante un cierto grado se pasa al catalán para este ámbito, sobretodo entre los hijos de la primera oleada migratoria. Este proceso ha sido largo y ha sido necesario esperar a la segunda generación para ver surgir un cambio. Por tanto, es pronto para que el fenómeno se manifieste en la nueva inmigración y para conocer cómo evolucionarán en ella los procesos de integración y cohesión generales. En este sendito, sus porcentajes de catalanización se reducen al 1,5\% entre los ecuatorianos y al $0 \%$ para los marroquíes, quienes si manifiestan algún cambio lo hacen hacia la castellanización o hacia el bilingüismo entre catalán y castellano.

De alguna manera los procesos de integración lingüística van paralelos a los que se dan con respecto a la integración con sus alteri. Es importante este ámbito familiar como comunidad de habla pues tiene una dosis importante como símbolo identitario y menos de carácter funcional o pragmático de otros ámbitos. 


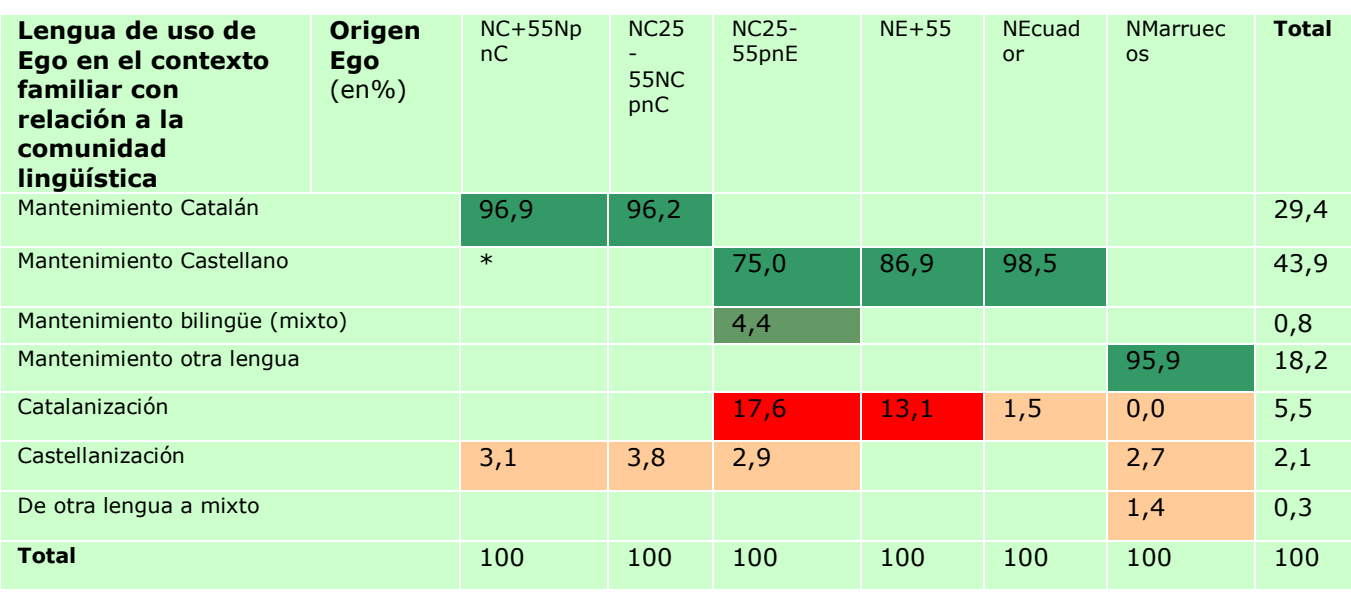

Tabla 4. Colectivo Ego y cambio de lengua en el contexto familiar.

*En blanco las situaciones o procesos que no se pueden dar por la naturaleza de las comunidades lingüísticas.

\section{En situación de contexto laboral}

La tabla 5 representa las tendencias en \% del comportamiento en el ámbito del trabajo entre los Egos entrevistados de diferentes colectivos o comunidades lingüísticas en su tránsito a comunidades de habla. Siguiendo la misma lógica que la tabla anterior, el mantenimiento en el habla se marca en azul oscuro y los procesos de cambio en rojo.

La tendencia del colectivo catalán es semejante a la precedente aunque se da una reducción del porcentaje no llegando al $80 \%$ en el mantenimiento del idioma. Pasa a ser más elevado el porcentaje de Egos que presentan castellanización en el habla: se trata de un $20 \%$ para los mayores de 55 años y de un $24 \%$ entre los 25 y 55 . El dato más llamativo se encuentra en el caso de los Egos NC25-55pNE: en más de la mitad de estos Egos, un 60\%, aparece un proceso de catalanización en el trabajo, porcentaje que también es importante en los NE+55: un 36\% participan de este proceso de catalanización en este contexto. Hecho que nos permite afirmar parte de nuestra hipótesis en relación al trabajo o ámbito laboral.

No obstante, con respecto a la segunda oleada de inmigración, mientras los ecuatorianos mantienen su lengua de origen en un $77 \%$ en el contexto laboral, un $63 \%$ de los marroquíes presentan tendencias de castellanización. Esto es, para la nueva inmigración la comunidad de habla en el trabajo es principalmente castellana. Siendo los porcentajes de catalanización, con respecto a estos colectivos, de un $22,7 \%$ para los ecuatorianos y de sólo un $2,7 \%$ para los marroquíes, quienes pasan a utilizar el castellano en este ámbito en un $63,0 \%$. 
Es de interés dar cuenta que dicha distinción en la emergencia de las comunidades de habla surgidas en el ámbito laboral puede vincularse al tipo de trabajo desempeñado para cada colectivo dentro del mercado de trabajo y a su polarización que, a su vez, se vincula con la necesidad de haber pasado o no por el sistema educativo catalán. En esta línea, en la tabla número 6 , se muestran los trabajos desempeñados para cada Ego de los distintos colectivos, manifestando que los tres primeros colectivos (NC+55NpnC, NC25-55NCpnC, NC25-55pnE) quienes presentan en mayor porcentaje un uso más considerable del catalán son los que desempeñan trabajos de mayor necesaria calificación académica (entiéndase profesionales y/o técnicos).

\begin{tabular}{|c|c|c|c|c|c|c|c|c|}
\hline $\begin{array}{l}\text { Lengua de uso de Ego } \\
\text { en el contexto laboral } \\
\text { con relación a la } \\
\text { comunidad lingüística }\end{array}$ & $\begin{array}{l}\text { Origen } \\
\text { Ego } \\
(\mathrm{en} \%)\end{array}$ & $\begin{array}{l}\mathrm{NC}+55 \\
\mathrm{NpnC}\end{array}$ & $\begin{array}{l}\text { NC25- } \\
55 \text { NCpnC }\end{array}$ & $\begin{array}{l}\text { NC25- } \\
55 p n E\end{array}$ & $\mathrm{NE}+55$ & NEcuador & NMarruecos & Total \\
\hline Mantenimiento Catalán & & 79,7 & 75,5 & & & & & 23,6 \\
\hline Mantenimiento Castellano & & * & & 33,8 & 63,9 & 77,3 & & 29,4 \\
\hline Mantenimiento bilingüe (mixto) & & & & 4,4 & & & & 0,8 \\
\hline Mantenimiento otra lengua & & & & & & & 16,4 & 3,1 \\
\hline Catalanización & & & & 60,3 & 36,1 & 22,7 & 2,7 & 20,8 \\
\hline Castellanización & & 20,3 & 24,5 & 1,5 & & & 63,0 & 19,0 \\
\hline De otra lengua a mixto & & & & & & & 17,8 & 3,4 \\
\hline Total & & 100 & 100 & 100 & 100 & 100 & 100 & 100 \\
\hline
\end{tabular}

Tabla 5. Origen Egos y cambio de lengua en el contexto laboral.

*En blanco las situaciones o procesos que no se pueden dar por la naturaleza de las comunidades lingüísticas.

\begin{tabular}{|c|c|c|c|c|c|c|}
\hline & $\mathrm{NC}+55 \mathrm{NpnC}$ & NC25-55NpnC & NC25-55NpnE & $\mathrm{NE}+55$ & NEcuador & NMarruecos \\
\hline $\begin{array}{l}\text { Empresario/a con asalariados } \\
\text { Empresario/a sin asalariados } \\
\text { Directores/as y jefes/as } \\
\text { Miembros de cooperativas } \\
\text { Profesionales, técnicos/as no } \\
\text { asalariados/as } \\
\text { Profesionales, técnicos/as } \\
\text { asalariados/as } \\
\text { Encargados/as, capataces } \\
\text { Trabajador/a cualificado } \\
\text { Trabajador/a sin especialización } \\
\text { Fuerzas armadas } \\
\text { No clasificable }\end{array}$ & $\begin{array}{c}6,3 \% \\
4,7 \% \\
1,6 \% \\
0,0 \% \\
1,6 \% \\
\\
32,8 \% \\
4,7 \% \\
23,4 \% \\
12,5 \% \\
0,0 \% \\
12,5 \%\end{array}$ & $\begin{array}{r}3,8 \% \\
0.0 \% \\
3,8 \% \\
1,9 \% \\
0,0 \% \\
\\
58,5 \% \\
1,9 \% \\
20,8 \% \\
1,5 \% \\
0,0 \% \\
1,9 \%\end{array}$ & $\begin{array}{c}0,0 \% \\
1,6 \% \\
4,8 \% \\
0,0 \% \\
1,6 \% \\
\\
42,2 \% \\
1,6 \% \\
25,8 \% \\
12,9 \% \\
0,0 \% \\
6,5 \%\end{array}$ & $\begin{array}{c}3,3 \% \\
0,0 \% \\
3,3 \% \\
0,0 \% \\
4,9 \% \\
24,6 \% \\
16 \% \\
20,2 \% \\
23.0 \% \\
1,6 \% \\
11,5 \%\end{array}$ & $\begin{array}{c}0,0 \% \\
0,0 \% \\
0,0 \% \\
0,0 \% \\
0,0 \% \\
\\
0,0 \% \\
0,0 \% \\
20,0 \% \\
60,0 \% \\
0,0 \% \\
20,0 \% \\
\end{array}$ & $\begin{array}{c}0,0 \% \\
1,4 \% \\
1,4 \% \\
0,0 \% \\
1,4 \% \\
\\
12,3 \% \\
0,0 \% \\
28,8 \% \\
37,0 \% \\
1,4 \% \\
16,4 \%\end{array}$ \\
\hline
\end{tabular}

Tabla 6. Origen de Ego y categoría socio-profesional.

\section{En situación de contexto de ocio y/o de relaciones de amistad}

La tabla 7 representa las tendencias en \% del comportamiento en el ámbito del ocio y/o relaciones de amistad entre los Egos entrevistados de los diferentes colectivos o comunidades lingüísticas en su tránsito a comunidades de habla. En este contexto que se brinda a que los vínculos e interacciones sean más espontáneos cabe esperar una tendencia semejante a la familiar. La lógica de los colores de la tabla es la misma que en las anteriores, siendo más oscuros los porcentajes de 
mantenimiento de la lengua de origen y marcando en rojo aquellos procesos de cambio.

Se observa efectivamente que los Egos de los colectivos de comunidades ligüísticas nacidos en Catalunya y los llegados de Ecuador no tienen tendencia a cambiar su lengua de origen para expresarse en las situaciones de habla. Ahora bien, se observa una diferencia interesante entre los NC+55pNC y los NC25-55pNC. Entre los segundos, nacidos en Catalunya, entre 25 y 55 años y de padres nacidos en Catalunya, un $28,3 \%$ habla en castellano con sus amigos en estos contextos de ocio, es decir, estos presentan una tendencia clara a la castellanización. Por el contrario, los primeros, que serían la generación de sus padres sólo llegan al 9,4\% de castellanización en el ámbito de ocio.

La misma tendencia pero con más intensidad puede observarse para el colectivo de castellanohablantes de origen o comunidades lingüísticas de los NE+55 y NC2555 pNE pero ahora con respecto a la catalanización: los NE+55 se catalanizan en estos contextos en un $26,2 \%$ y los NC25-55pNE en un $39,7 \%$.

\begin{tabular}{|c|c|c|c|c|c|c|c|c|}
\hline $\begin{array}{l}\text { Lengua de uso de } \\
\text { Ego en el contexto } \\
\text { ocio con relación a la } \\
\text { comunidad } \\
\text { lingüística }\end{array}$ & $\begin{array}{l}\text { Origen } \\
\text { Ego } \\
(\mathrm{en} \%)\end{array}$ & $\begin{array}{l}\mathrm{NC}+5 \\
5 \mathrm{Npn} \\
\mathrm{C}\end{array}$ & $\begin{array}{l}\mathrm{NC} 25 \\
- \\
55 \mathrm{NC} \\
\mathrm{pnC}\end{array}$ & $\begin{array}{l}\text { NC25 } \\
- \\
55 p n \\
E\end{array}$ & $\begin{array}{c}\mathrm{NE}+5 \\
5\end{array}$ & NEcuador & $\begin{array}{c}\text { NMarrue } \\
\text { cos }\end{array}$ & Total \\
\hline \multicolumn{2}{|l|}{ Mantenimiento Catalán } & 90,6 & 71,7 & & & & & 23,6 \\
\hline \multicolumn{2}{|l|}{ Mantenimiento Castellano } & $*$ & & 52,9 & 73,8 & 93,9 & & 29,4 \\
\hline \multicolumn{2}{|c|}{ Mantenimiento bilingüe (mixto) } & & & 5,9 & & & & 0,8 \\
\hline \multicolumn{2}{|c|}{ Mantenimiento otra lengua } & & & & & & 56,2 & 3,1 \\
\hline \multicolumn{2}{|l|}{ Catalanización } & & & 39,7 & 26,2 & 6,1 & 2,7 & 20,8 \\
\hline \multicolumn{2}{|l|}{ Castellanización } & 9,4 & 28,3 & 1,5 & & & 30,1 & 19,0 \\
\hline \multicolumn{2}{|l|}{ De otra lengua a mixto } & & & & & & 11,0 & 3,4 \\
\hline \multicolumn{2}{|l|}{ Total } & 100 & 100 & 100 & 100 & 100 & 100 & 100 \\
\hline
\end{tabular}

Tabla 7. Origen Egos y cambio de lengua en el contexto de amistad y ocio.

*En blanco las situaciones o procesos que no se pueden dar por la naturaleza de las comunidades lingüísticas.

Haciendo un zoom de la tabla anterior, en la tabla 8 se cruzan las dos comunidades lingüísticas para ver su trasferencia hacia comunidades de habla en sus lenguas invertidas.

\begin{tabular}{|l|l|l|l|l|}
\hline & NC+55pNC & NC25-55pNC & NE+55 & NC25-55pNE \\
\hline Catalanización & & & $26,2 \%$ & $39,7 \%$ \\
\hline Castellanización & $\mathbf{9 , 4} \%$ & $\mathbf{2 8 , 3} \%$ & & \\
\hline
\end{tabular}

Tabla 8. Trasferencias de comunidades lingüísticas a comunidades de habla en el contexto de actividades de ocio y relaciones de amistad.

Aparece un doble fenómeno, por el primero, las generaciones más jóvenes de ambas comunidades lingüísticas son más proclives a dicha inversión, esto es, al uso 
de la lengua de la otra comunidad como comunidad de habla en este contexto; el segundo, la comunidad lingüística en castellano es más proclive que la catalana a dichos trasvases invertidos. Es decir, que al menos en el dominio del uso de la lengua y en dicho ámbito del ocio y relaciones de amistad, los más jóvenes y los de habla castellana de origen tienden a una mayor integración lingüística que los otros colectivos. Lo que va en la dirección ya considerada acerca de las relaciones de cohesión e integración que mantienen las comunidades lingüísticas con los alteri por razones de conocimiento y comunicación.

En referencia a los marroquíes más de la mitad de sus Egos, un $56,2 \%$, hablan en árabe (señalada con otra en la tabla) en sus interacciones con los amigos. Y, los que no lo hacen, utilizan en gran medida el castellano, un 30,1\%, y menos el catalán, un $2,7 \%$. Los ecuatorianos lo hacen un $30.1 \%$ en castellano y sólo un $6,1 \%$ entran en la catalanización.

Por lo tanto, como era de esperar los límites de las comunidades lingüísticas se difuminan en contextos menos formales o de generación espontánea como es el ocio o con las amistades, manifestándose la emergencia de dos comunidades de habla según situaciones o amistades y promoviendo una InL que no se ve sólo en una misma dirección.

Retomando las hipótesis de investigación cabe apuntar que:

- Considerando como colectivos base las comunidades lingüísticas, hemos revisado como los Egos aquí encuestados presentan gran tendencia cohesiva en sus relaciones con los Alteri. No obstante la integración se da hasta cierto punto sobretodo para los Egos nacidos en el resto de España y, con mayor fuerza, entre sus hijos potenciales.

- En relación a los procesos de cambio en el habla según los contextos o momentos de interacción hemos revisado que los Egos aquí encuestados mantienen su lengua de origen, esto es, la lengua de su comunidad lingüística en el ámbito familiar, siendo, otra vez las generaciones de la primer oleada migratoria los que presentan mayor cambio de la lengua en el ámbito familiar.

- Para el ámbito laboral hemos visto que la tendencia es de división o polarización, mientras que los colectivos con trabajos más estables y con requerimiento académico presentan o bien mantenimiento hacia el catalán o bien cierta catalanización, para los colectivos de la nueva inmigración la 
tendencia se presenta a la inversa, manteniéndose en el castellano o "castellanizándose".

- Y, finalmente, en el ámbito menos formal y pautado, parece ser que las comunidades lingüísticas de origen se difuminan gracias, seguramente, al bilingüismo de buena parte de los colectivos tanto en la dirección de la "catalanización" como en la de la "castellanización". No obstante, la nueva oleada migratoria presenta mayor tendencia a la castellanización o al mantenimiento del castellano, va a ser necesario esperar las nuevas generaciones para ver como los mecanismos de CoL e InL se manifiestan.

\section{Conclusiones}

Es necesario recordar que el objetivo de este artículo consiste en estudiar el uso y los procesos que diferentes colectivos de comunidades lingüísticas llevan a cabo en el uso de las lenguas, como formas de cohesión y/o integración lingüística y ello también en diferentes situaciones o contextos como modos de identificarlas en tanto que comunidades de habla. Para ello hemos partido de la distinción entre comunidad lingüística como un colectivo con idéntica lengua de origen y comunidad de habla como un colectivo que usa lengua en determinas situaciones, momentos, instituciones o genéricamente espacios o ámbito sociales. Dentro de este objetivo general nos ha interesado examinar más detenidamente las diferencias entre colectivos, los procesos de trasferencia en el cambio de lengua según contextos y la homología que se da entre los fenómenos lingüísticos y los de cohesión e integración de los colectivos con sus respectivos alteri de conocidos y personas con las que se comunican.

Con todo, se trata de un estudio preliminar utilizando un número mínimo de variables y situaciones; además de no ser exhaustivo para todas las comunidades lingüísticas de Catalunya. Pero cabe dar cuenta que buena parte del artículo ha ido en la dirección de definición y precisión de los conceptos de cohesión e integración lingüística. Por otro lado el proceso de análisis ha sido simple, primero examinando la cohesión e integración de cada comunidad lingüística con relación a sus alteri de conocidos y de trato comunicativo regular. En segundo lugar, analizando el comportamiento de los Egos de los colectivos de las diferentes comunidades lingüísticas, de las que conocemos su comportamiento cohesivo e integrativo, como comunidades de habla en diferentes contextos.

Veamos algunas conclusiones generales de dichos fenómenos y procesos; los supuestos y conclusiones más específicos se han dado cuenta ya con un cierto detalle. 
Hay un grado de paralelismo entre la $\operatorname{CoS}$ e InS de las comunidades lingüísticas desde un punto de vista relacional y comunicativo con los conocidos con los que se comunican independientemente de la lengua y la cohesión e integración específicamente lingüística, manifestado en la tendencia mayoritaria a que una comunidad lingüística conserve su lengua en diferentes situaciones como manifestación de cohesión. Con todo también aparezen fenómenos de trasferencia invertida a las lenguas que no son de origen en situaciones diferentes lo que venimos llamando catalanización o castellanización.

Este fenómeno general integrativo de traslación de comunidad lingüística a comunidad de lengua invirtiendo las lenguas propias es importante, si bien no es mayoritario pues lo es el de cohesión lingüística. En esta traslación invertida de lenguas en determinadas situaciones, o integración lingüística, está más presente la generación entre 25 y 55, la comunidad lingüística en castellano y la primera ola migratoria. En mucha menor medida en las comunidades de ecuatorianos y marroquíes.

En el contexto familiar la tendencia general es la de mantener la lengua de origen, pues se trata de un espacio identitario y del que no es necesario ni, probablemente, deseado perder la cohesión grupal. Con respecto al contexto laboral una buena mayoría de "hijos de la primera ola de inmigración" tienen la tendencia a la catalanización. En este sentido el sistema educativo y/o la misma trayectoria profesional tienen seguramente algo que ver. En los contextos de ocio y amistad la tendencia es más a la castellanización.

Se observan también las diferencias existentes según los contextos en los que operan o se configuran las comunidades de habla. Las cohesión lingüística es mayor de las comunidades lingüísticas hacia su propia lengua en contextos familiares que en los otros dos, más instrumental en la de trabajo y más expresivo en la de amistad y ocio. En estos ámbitos se da mayor integración que en el familiar, probablemente por razones de mayor utilidad o de afinidades, si bien igualmente es más elevada la cohesión lingüística. En todo este proceso, el bilingüismo de hecho y el formativo así como la presencia necesariamente simultánea de personas de diferentes comunidades lingüísticas en los mismos ámbitos son factores primordiales que sería interesante seguir revisando.

\section{Bibliografía}

Almedia, A. (1999). Sociolingüística. Sevicio de publicaciones: Universidad de la Laguna. 
Bernard, P. (1999). Social Cohesion: A Dialectical Critique of a Quasi-Concept?, Ottawa: Strategic Research and Analysis Directorate, Department of Canadian Heritage.

Bloomfield, L. (1983). An Introduction to the study of language. Amsterdam: John Benjamins.

Bourdieu, P. (1986). - The Forms of Capital, en Richards J.G. (ed.), Handbook of Theory and Research for the Sociology of Education, New York: Greenwood Press, pp. 241-258.

Brehm, J.; Rahn W. (1997). Individual-Level Evidence for the Causes and Consequences of Social Capital, American Journal of Political Science 41:999-1023

Burt, R.S. (1992). Structural Holes: The Social Structure of Competition, Cambridge, MA:Harvard University Press.

Cantle, T. (2005). Community Cohesion: A New Framework for Race and Diversity, Basingstoke: Palgrave Macmillan.

Coleman, J. (1990). Foundations of Social Theory, Cambridge, MA: Harvard University Press.

Duranti, A. (1997). Linguistic anthropology. Cambridge, Cambridge University Press.

Fukuyama, F. (1995). Trust, the social virtues and the creation of prosperity. London: Penguin Books.

Fukuyama, F. (1997). - Social capital and the modern capitalist economy: Creating a high trust workplace, Stern Busi-ness Magazine, 4(1).

Granovetter, M. (1973). "The strength of weak ties", American Journal of Sociology, no 78: $1360-1380$.

Gross, N.; Martin W.E. (1952). "On Group Cohesiveness", American Journal of Sociology, 52: 546-54.

Gualda, Caballero, E. (2007) Integración Social de los inmigrantes y modelos teóricos que la explican, Universidad de Huelva, España.

Gumperz, J. (1968). «The speech community». En International Encyclopedia of the Social Sciences. Londres: Macmillan, 381-386.

Gumperz, J. y Hymes, D. (1972). Directions in Sociolinguistics: The Ethnography of Communication. New York: Holt, Rinehart \& Winston. 
Halliday, M.A.K. (1982). El lenguaje como semiótica social: la interpretación social del lenguaje y del significado. Mèxic: Fondo de Cultura Económica, cop.1982.

Hudson, R.A (1981). La Sociolingüística. Barcelona: Anagrama.

Labov, W. (1972). Language in the Inner City: Studies in the Black English Vernacular. Philadelphia: University of Pennsylvania Press.

Lopez Roldán P.; Lozares C. (2008b). "La construcción de la muestra. El trabajo de campo de la Encuesta de condiciones de vida y hábitos de la población de Catalunya" Metodologies i Recerques. IERMB, Barcelona, no 1:17-39.

Lozares, C., (1996). - La teoría de redes sociales-, Papers, nº48.

Lozares, Carlos; Verd, Joan Miquel; López-Roldán, Pedro; Martí, Joel; Molina, Jose Luis (2011). "Cohesión, Vinculación e Integración sociales como formas de Capital social", REDES-Revista hispana para el análisis de redes sociales, vol. 20 \#1 (http://revista-redes.rediris.es)

Lozares, Carlos; López-Roldan, Pedro; Verd, Joan Miquel; Marti, Joel Marti;Molina, José Luis; Bolíbar, Mireia; Cruz, Irene. (2011). "El análisis de la Cohesión, Vinculación e Integración sociales en las encuestas Ego-net", REDES-Revista hispana para el análisis de redes sociales, vol. 20 \#3 (http://revistaredes.rediris.es)

Marbà, T. (1987). Qüestions obertes en didàctia de la llengua. Barcelona: Universitat de Barcelona. Departament de didáctica de la llengua i literatura.

Mollà, T. (2002). Llengües globals, llengües locals. Alzira: Bromera.

Moody, J.; White D .R (2003). - Social Cohesion and Embeddedness: A hierarchical conception of social groups, American Sociological Review, 68:107-127

Narayan, D. (1999). Bonds and Bridges: Social capital and poverty, Word Bank, Washington DC.

Pitarch, V. (1984). Reflexió crítica sobre la "llei d'ús i ensenyament del valencià". Valéncia: Eliseu Ciment.

Portes, A. (1998). - Social capital: Its origins and application in modern sociology, Annual Review of Sociology, 24, 1-24.

Putnam, R. (1993). - The prosperous community: social capital and public life, The American Prospect, 13.

Putnam, R. (2001). - Le déclin du capital social aux Etats-Unis. Entretien avec Robert Putnam\|, Sciences Humaines 33: 63. 
Putnam, R. D. (1995). -Bowling alone: America's declining social capitalll, Journal of Democracy, 6(1):65-78.

Serrano, S. (1993). Comunicació, societat i llenguatge. Barcelona: Editorial Empuries.

Weinreich, U. (1974). Lenguas en contacto: descubrimientos y problemas. Venezuela: Universidad Central de Venezuela. 